\title{
Parental Quality of Life and Involvement in Intervention for Children or Adolescents with Autism Spectrum Disorders: A Systematic Review
}

\author{
Alessandro Musetti 1,*(D), Tommaso Manari ${ }^{1} \mathbb{D}$, Barbara Dioni ${ }^{1,2}$, Cinzia Raffin ${ }^{2}$, Giulia Bravo ${ }^{2} \mathbb{D}$, \\ Rachele Mariani $^{3}{ }^{(D)}$, Gianluca Esposito ${ }^{4,5,6}{ }^{(D}$, Dagmara Dimitriou ${ }^{7,8}{ }^{(D}$, Giuseppe Plazzi ${ }^{9,10}$, \\ Christian Franceschini ${ }^{11}$ (D) and Paola Corsano ${ }^{1}$
}

Citation: Musetti, A.; Manari, T.; Dioni, B.; Raffin, C.; Bravo, G.;

Mariani, R.; Esposito, G.;

Dimitriou, D.; Plazzi, G.; Franceschini, C.; et al. Parental Quality of Life and Involvement in Intervention for Children or

Adolescents with Autism Spectrum Disorders: A Systematic Review. J. Pers. Med. 2021, 11, 894. https:// doi.org/10.3390/jpm11090894

Academic Editors: Richard E. Frye, Richard Boles, Shannon Rose and Daniel Rossignol

Received: 4 August 2021

Accepted: 6 September 2021

Published: 8 September 2021

Publisher's Note: MDPI stays neutral with regard to jurisdictional claims in published maps and institutional affiliations.

Copyright: (c) 2021 by the authors. Licensee MDPI, Basel, Switzerland. This article is an open access article distributed under the terms and conditions of the Creative Commons Attribution (CC BY) license (https:/ / creativecommons.org/licenses/by/ $4.0 /)$.
1 Department of Humanities, Social Sciences and Cultural Industries, University of Parma, Borgo Carissimi 10, 43121 Parma, Italy; tommaso.manari@unipr.it (T.M.); barbara.dioni@unipr.it (B.D.); paola.corsano@unipr.it (P.C.)

2 Fondazione Bambini e Autismo Onlus, 33170 Pordenone, Italy; c.raffin@bambinieautismo.org (C.R.); g.bravo@bambinieautismo.org (G.B.)

3 Department of Dynamic and Clinical Psychology, Sapienza University of Rome, 00185 Rome, Italy; rachele.mariani@uniroma1.it

4 Social and Affective Neuroscience Lab, Psychology Program-SSS, Nanyang Technological University, Singapore 639818, Singapore; gianluca.esposito@unitn.it

5 Lee Kong Chian School of Medicine, Nanyang Technological University, Singapore 636921, Singapore

6 Affiliative Behaviour and Physiology Lab, Department of Psychology and Cognitive Science, University Trento, 38068 Rovereto, Italy

7 Sleep Education and Research Laboratory, Department of Psychology and Human Development, UCL-Institute of Education, London WC1H 0AA, UK; d.dimitriou@ucl.ac.uk

8 The National Institute for Stress, Anxiety, Depression and Behavioural Change (NISAD), 25221 Helsingborg, Sweden

9 Department of Biomedical, Metabolic and Neural Sciences, University of Modena and Reggio Emilia, 41125 Modena, Italy; giuseppe.plazzi@unimore.it

10 IRCCS Institute of Neurological Sciences of Bologna (ISNB), 40139 Bologna, Italy

11 Department of Medicine and Surgery, University of Parma, 43121 Parma, Italy;

Christian.franceschini@unipr.it

* Correspondence: alessandro.musetti@unipr.it; Tel.: +39-0521-034820

\begin{abstract}
Previous research has examined several parental, child-related, and contextual factors associated with parental quality of life (QoL) among parents with a child or an adolescent with autism spectrum disorders (ASD); however, no systematic review has examined the relationship between parental QoL and parental involvement in intervention. To fill this gap, a systematic review was conducted using four electronic databases and checked reference lists of retrieved studies. Records were included in the systematic review if they presented original data, assessed parental QoL, and involvement in intervention for children or adolescents with ASD, were published in peer-reviewed journals between 2000 and 2020, and were written in English. Among the 96 screened full-texts, 17 articles met the eligibility criteria. The selected studies included over 2000 parents of children or adolescents with ASD. Three categories of parental involvement (i.e., none, indirect, direct) were identified, which varied across studies, although most had direct parental involvement. The results from this review show that increased parental involvement in the intervention for children or adolescents with ASD may be one way to promote their QoL. However, further research specifically focused on parental involvement during the intervention for children and adolescents with ASD is warranted.
\end{abstract}

Keywords: autism spectrum disorder; quality of life; parents; intervention; systematic review 


\section{Introduction}

Autism spectrum disorder (ASD) is a pervasive neurodevelopmental disorder characterised by persistent atypicalities in social communication and social interactions across different domains, together with restricted, repetitive, stereotyped patterns of behaviour, interest, or activities [1]. Although there is large variability in the expression of ASD symptoms, many individuals with ASD need lifelong assistance in daily life that is usually provided by family members, especially parents [2]. Therefore, the presence of a family member with ASD features such as challenging behaviours [3,4] or sleep problems [5,6] can significantly affect and challenge the entire family system [7], with a mostly negative impact on the quality of life (QoL) and on the relationship quality of closer family members such as siblings [8,9] and parents $[10,11]$.

Although a lot of research has been conducted to examine the relationship between the characteristics of offspring with ASD, distress and psychological difficulties of family members [12,13], less attention has been paid to the impact of the characteristics of the intervention on family functioning [14,15]. Moreover, most of the research on interventions for ASD has so far been focused on child outcomes, disregarding the impact on parents [14]. This is surprising, given the increasing parental involvement in activities of children and adolescents with ASD [16,17] including the intervention process [18]. In many cases, parental involvement in intervention covers a broad range of activities, from parent training and homework routines, even participation in intervention design and implementation [19]. Therefore, taking into account the family outcomes, it would be possible to gain a more comprehensive view of the effectiveness of an intervention and, in turn, better shape intervention design and implementation [14,20,21]. Most early research in this field unilaterally focused on the negative outcomes (e.g., parental stress) of having a child with ASD [22]. However, a growing number of studies have investigated parental QoL in an endeavour to attempt to capture the variability of parental adaptation and to more deeply comprehend difficulties faced by these parents $[23,24]$. This interesting broadening of perspective highlights how some parents can cope with the stress resulting from caring for their children with ASD, and in turn, learn and improve their competencies in the process $[25,26]$. For example, when mothers are able to gain emotional resolution on their child's diagnosis of ASD, they tend to develop a more supportive parenting style [27]. QoL is a multidimensional and wide-ranging construct, characterized by the individual's emotional, physical, and financial well-being, interpersonal relationships, goals, expectations and concerns, and their interactions with salient features of the environment [28,29]. According to the World Health Organization (WHO) Quality of Life Assessment Group [30], QoL is defined as an "individual's perception of their position in life in the context of the culture and value systems in which they live, and in relation to their goals, expectations, standards, and concerns" (p. 11). Up to date, only one study [24] has systematically reviewed published literature about the parental QoL of children and adolescents with ASD. Thus far, several variables have been found to be associated with lower QoL among parents of children and adolescents with ASD including parental characteristics (e.g., being a mother, parental mental health problems, maladaptive parental coping strategies, and low parental self-efficacy), child characteristics (e.g., child behavioural problems, child emotional problems, ASD severity, and child's age), and contextual factors (e.g., low employment status, low household income, low availability of social and professional support, and lack of participation in health promoting activities). However, no systematic review exists that summarizes the currently available evidence on the relationship between parental QoL and parental involvement in intervention for their children or adolescents with ASD. This constitutes a relevant gap in the literature considering the recent increased emphasis on family-focused versus professional-driven interventions [14,31], which the present study intends to fill. Specifically, a previous systematic review on parental QoL of children of ASD by Vasilopoulou et al. [24] revealed a need for greater focus on parents to provide tailored intervention for this population. The current review aimed to identify 
and discuss the role of parental involvement in intervention in relation to QoL among the parents of children or adolescents with ASD.

\section{Methods}

\subsection{Protocol and Registration}

The present systematic review adhered to the Preferred Reporting Items for Systematic Reviews and Meta-Analyses (PRISMA) statement [32]. The protocol was registered at the International Prospective Register of Systematic Reviews (PROSPERO) data repository in February 2021 (registration code: CRD42021230103).

\subsection{Study Selection}

The selection process identified the following eligibility criteria. Inclusion criteria (IC): (IC1) studies had to be focused on parents of children and adolescents (i.e., $<21$ years old) with a formal diagnosis of ASD; (IC2) they had to contain quantitative, qualitative, or mixed methods approaches; and (IC3) be published between 2000 and 2020 in (IC4) peer-reviewed journals and written in English. We rejected articles that met one of the following excluding criteria (EX): (EX1) case reports, commentaries, editorials, meeting abstracts were not considered; (EX2) other review articles were consulted but not included finally and to narrow our search procedure, and we excluded (EX3) studies that dealt with the quality of life of parents of children with disabilities different from ASD.

A systematic search was carried out in January 2021, in four online databases: Scopus, Web of Science, PubMed, and PsycINFO. To reduce the risk of methodological biases, no filters were applied in the preliminary searches. We combined the selected keywords with the Boolean operators AND/OR in the following order: ("parent") AND ("ASD") OR ("autis") AND ("quality") AND ("life") AND ("intervent"), in Titles, Abstracts, and Keywords.

All references collected through database searches were exported to the systematic reviews web application Rayyan (https:/ / rayyan.qcri.org/ (accessed on 1 August 2021)). Duplicate records were ruled out, then the titles and abstracts of all remaining articles were independently screened. The use of multiple reviewers may reduce the risk of rejecting relevant reports, as noted by Edwards and colleagues [33]. Results were then compared and in the case of disagreement, the conflicting choices were inspected and discussed further until a consensus was reached. The second step included the screening of the full-texts of the chosen studies, in order to select those where the quality of life was a specified outcome. Reference lists of relevant studies and reviews were examined for additional pertinent records. The articles deemed eligible by all reviewers and that met all the inclusion criteria were included for the final screening, and those that met at least one exclusion criteria were formally excluded with reasons.

\subsection{Quality Assessment}

The critical assessment of each paper was conducted with AXIS, a quality assessment tool for observational and cross-sectional studies [34]. It comprises a checklist of 20 items to evaluate the overall study design as well as the risk of bias. The items were scored as follows: Yes $=1$, No and Don't know $=0$, and resulted in a final score that ranged from 0 to 20, with higher scores indicating a higher assessed quality. Adopting the classification used in other literature reviews (e.g., [35]), we further distinguished three quality ratings: low quality ( $0-7$ points), moderate quality ( $8-14$ points), and high quality (15-20 points). The qualitative assessment outcome is discussed in the Results section.

\section{Results}

\subsection{Study Selection}

The systematic search was first performed in four online databases: Scopus, Web of Science, PubMed, and PsycINFO, and yielded a total of 1217 records. Duplicate articles were removed with the reference manager Mendeley desktop (https:/ /www.mendeley. 
com/ (accessed on 1 August 2021)) and resulted in 808 unique references, which were imported to the systematic reviews web application Rayyan (https:/ / rayyan.qcri.org/ (accessed on 1 August 2021)). Titles and abstracts were then independently screened for potentially relevant papers, resulting in 96 eligible references and 712 excluded because they did not meet the predefined inclusion criteria. As the last step, 79 full-texts were excluded with reasons (e.g., non-pertinent, non-pertinent outcome, no empirical data, or without intervention for children with ASD) and 17 articles were found to be appropriate for the qualitative synthesis. The four steps defined in the PRISMA procedure (i.e., Identification, Screening, Eligibility, and Inclusion) are represented in Figure 1 and the excluded articles are listed in Supplementary Table S1.
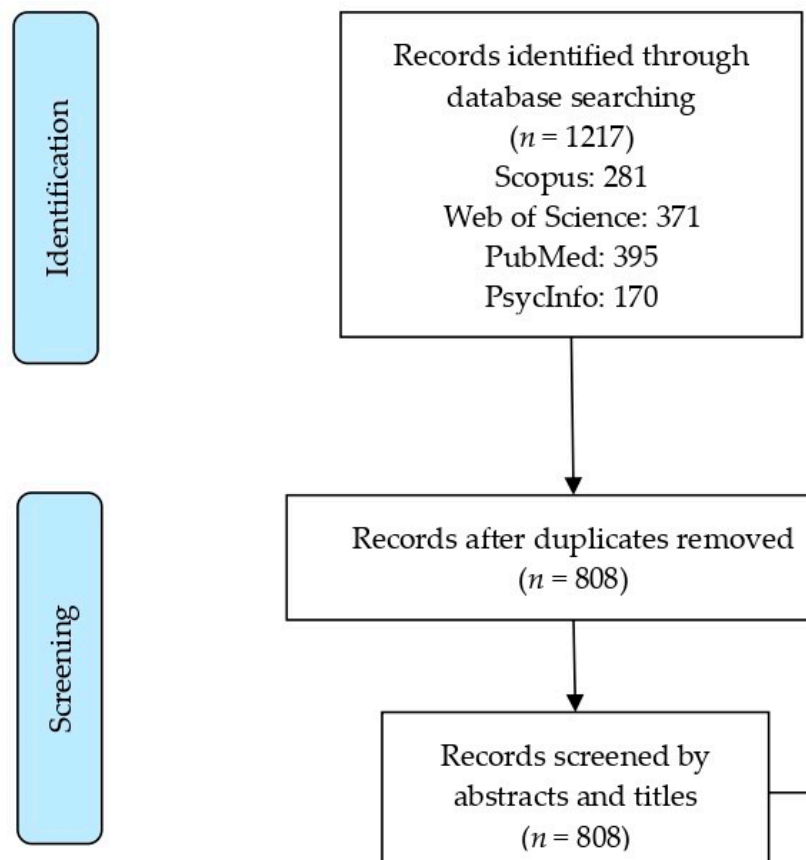

Records after duplicates removed $(n=808)$
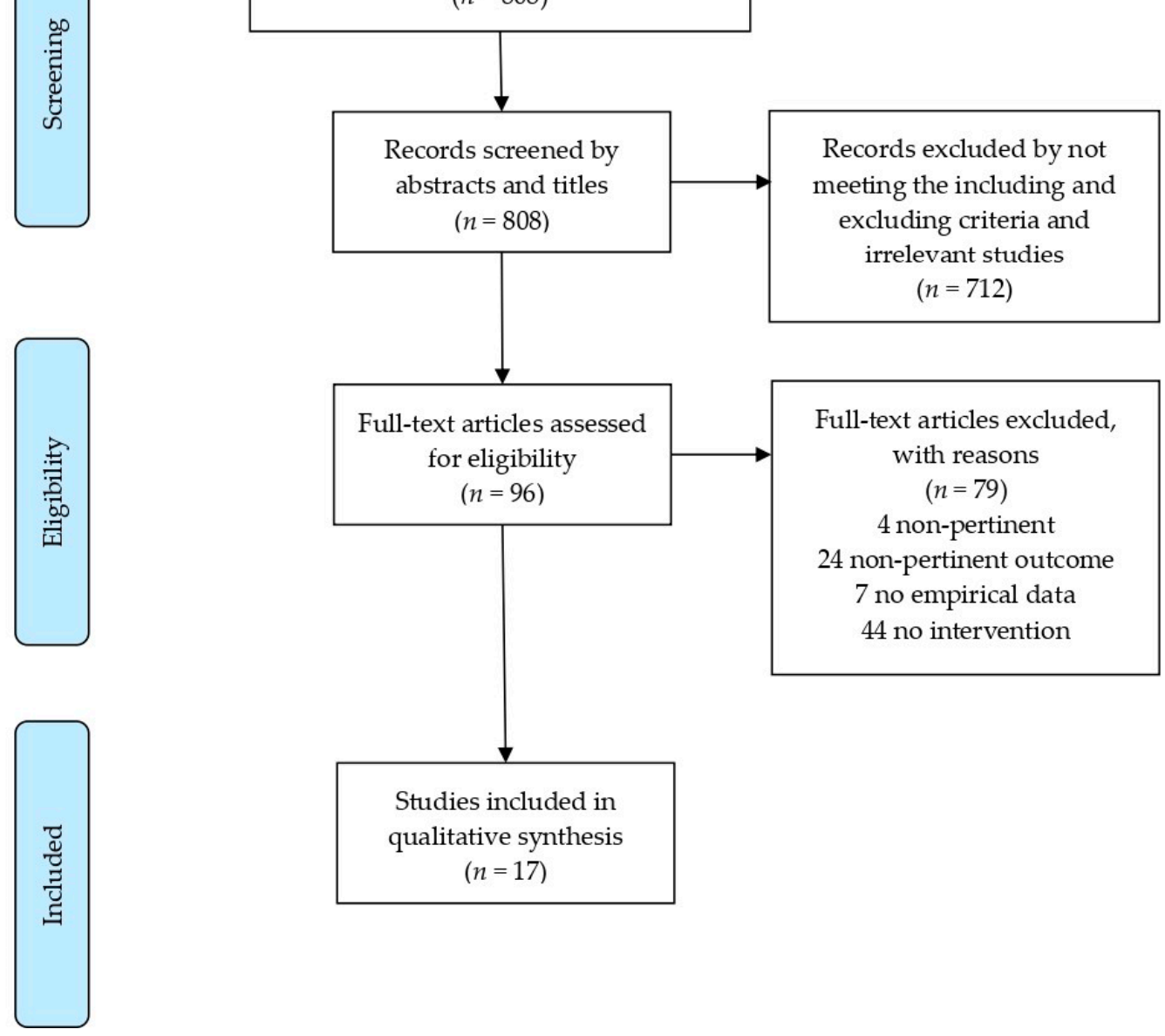

Studies included in qualitative synthesis $(n=17)$

Figure 1. Flow diagram of the search strategy. Modified from the Preferred Reporting Items for Systematic reviews and Meta-Analyses statement flow diagram [32]. 


\subsection{Study Characteristics}

A summary of the information extracted from each study is presented in Table 1 . The present systematic review examined 17 studies that were published between 2011 and 2020 in peer-reviewed journals that focused on the intrafamilial QoL of parents with a child or an adolescent affected by ASD. The adopted methodological designs were mixed and included cross-sectional designs, qualitative and observational studies, randomized controlled trials, and a validation study.

The reviewed studies involved a total of 1965 children and adolescents and 2040 parents or primary caregivers (partial information was available from three studies). The age of the offspring ranged from 2 to 20 years old $(M=7.43, S D=3.22)$, and $74.48 \%$ of the involved participants were female (mothers or female caregivers; partial information was available from three studies).

In relation to the geographical distribution (i.e., where the study was conducted and the data was collected), the 17 studies were carried out in Europe ( $n=8$, United Kingdom, France, Sweden), North America ( $n=2$, United States, Canada), Asia ( $n=2$, China, Taiwan), and Australia $(n=5)$.

The present systematic review was focused on parenting education programmes and interventions. Similarly to previous studies (e.g., [36]), we distinguished between three levels of parental involvement: none (i.e., parents are not involved in intervention design and implementation), indirect (i.e., parents are involved in secondary forms of interventions such as parent training or homework routines), and direct (i.e., parents are actively involved in primary intervention development and implementation).

Accordingly, we identified a heterogeneous number of studies that included no $(n=2)$, indirect $(n=3)$, or direct $(n=12)$ levels of parental involvement. The interventions were performed in clinical settings $(n=7)$, at home $(n=4)$, or a combination of the two (i.e., mixed settings, $\mathrm{n}=3$; information not available for three records).

The construct dimensions related to the parental QoL were assessed with multiple instruments (see Table 2) including the Family Quality of Life Scale [37], the Parenting Stress Index [38], and the WHOQOL-BREF [28]. Since different studies implemented qualitative and observational approaches, semi-structured interviews, questionnaires, and ad-hoc rating scales were also used. 
Table 1. Characteristics of the reviewed studies $(n=17)$.

\begin{tabular}{|c|c|c|c|c|c|c|c|c|c|}
\hline $\begin{array}{l}\text { Authors } \\
\text { (Year) }\end{array}$ & Location & Study Design & $\begin{array}{c}\text { Sample Characteristics } N \\
\text { Age }=M(S D)\end{array}$ & $\begin{array}{c}\text { Parental } \\
\text { Involvement }\end{array}$ & Intervention & Setting & Length/Frequency & Instruments & Relevant Findings \\
\hline $\begin{array}{c}\text { Aandersson } \\
\text { et al. (2017) } \\
\text { [39] }\end{array}$ & Sweden & Qualitative study & $\begin{array}{c}56 \text { Parents of Children with } \\
\text { ASD }(n=56 \text { Children; } \\
M_{\text {age }} \approx 9 \text { Years, } \\
\text { Female }=23.21 \%)\end{array}$ & Indirect & Early Intervention, ABA & Clinics & 2 Years $/ 25$ h/Week & $\begin{array}{l}\text { Semi-Structured } \\
\text { Questionnaire }\end{array}$ & $\begin{array}{l}\text { Thematic Content Analysis } \\
\text { Too much responsivity and lack of } \\
\text { knowledge about intervention } \\
\text { methods. Perceived support as } \\
\text { unequal, uncoordinated, and with } \\
\text { variations. Unequal treatments } \\
\text { depending on socioeconomic } \\
\text { status. lack of individualization } \\
\text { of interventions. }\end{array}$ \\
\hline $\begin{array}{l}\text { Baghdadli } \\
\text { et al. (2014) } \\
\text { [10] }\end{array}$ & France & Cross-Sectional & $\begin{array}{c}152 \text { Mothers }(92.12 \%) \text { and } \\
13 \text { Fathers of Adolescents } \\
\text { with ASD } \\
(n=152 \text { Adolescents; } \\
M_{\text {age }}=15 \text { Years; } S D=1.6 \\
\text { Female }=17.8 \%)\end{array}$ & None & Mixed & Clinics & NA/31.3 $5 \mathrm{~h} /$ week & Par-DD-QoL & $\begin{array}{l}\text { Polytomic Logistic Regression } \\
\text { Analysis } \\
\text { A higher number of hours of } \\
\text { specialized intervention is } \\
\text { associated with lower parental } \\
\text { emotional QoL (ORa = 2.69, } \\
\text { 95\% CI = 1.1-5.9, } p=0.04) .\end{array}$ \\
\hline $\begin{array}{l}\text { Derguy et al. } \\
\text { (2018) [40] }\end{array}$ & France & Cross-Sectional & $\begin{array}{c}115 \text { Parents } \\
\text { (Female }=63.5 \%) \text { of } \\
\text { Children with ASD } \\
\left(n=78 \text { Children; } M_{\text {age }}=6.3,\right. \\
S D=2.3, \text { Female }=23.1 \%)\end{array}$ & None & $\begin{array}{l}\text { Psycho-Educational } \\
\text { Intervention (74\% of the } \\
\text { Sample) }\end{array}$ & NA & NA & WHOQOL-BREF & $\begin{array}{l}\text { Hierarchical Regression Analyses } \\
\text { Parents showed better QoL } \\
\text { whether their child received } \\
\text { psychoeducation intervention } \\
\quad(\beta=0.25, p=0.010)\end{array}$ \\
\hline $\begin{array}{l}\text { Due et al. } \\
\text { (2018) [41] }\end{array}$ & Australia & $\begin{array}{l}\text { Mixed-Method } \\
\text { Study }\end{array}$ & $\begin{array}{c}27 \text { Parents } \\
\text { (Female }=48.14 \%) \text { of } \\
\text { Children with ASD } \\
\left(n=27 \text { Children; } M_{\text {age }}=5,\right. \\
S D=2.0, \text { Female }=18.52 \%)\end{array}$ & Direct & Early intervention & Clinics & NA & $\begin{array}{l}\text { Quality of Life in } \\
\text { Autism } \\
\text { Questionnaire; } \\
\text { Semi-Structured } \\
\text { Interviews }\end{array}$ & $\begin{array}{l}\text { Thematic Content Analysis } \\
\text { Parental direct involvement in the } \\
\text { intervention increased several } \\
\text { aspects of their QoL (e.g., sense of } \\
\text { competence and confidence as } \\
\text { parents, community participation). } \\
\text { Paired Sample Wilcoxon Signed }\end{array}$ \\
\hline $\begin{array}{l}\text { Hwang et al. } \\
\text { (2015) [42] }\end{array}$ & Taiwan & Pre-Post Design & $\begin{array}{l}\text { 6 Mother-Child dyads } \\
\text { (Children with ASD; } \\
\text { Age Range }=8-15 \text { Years, } \\
\text { Female }=20 \% \text { ) }\end{array}$ & Direct & $\begin{array}{l}\text { Parent-Mediated Home-Based } \\
\text { Training }\end{array}$ & Home & 12 Months & $\begin{array}{l}\text { Family Quality of } \\
\text { Life (FQoL) }\end{array}$ & $\begin{array}{l}\text { Rank Test } \\
\text { The parent-mediated home-based } \\
\text { training was associated with } \\
\text { marginally significant increase in } \\
\text { family quality of life. }\end{array}$ \\
\hline $\begin{array}{l}\text { Ji et al. (2014) } \\
\quad[43]\end{array}$ & China & $\begin{array}{l}\text { Quasi-Experimental } \\
\text { Design }\end{array}$ & $\begin{array}{c}22 \text { Caregivers } \\
\text { (Female }=90.9 \% \text { ) of } \\
\text { Children with ASD } \\
\text { (Intervention Group, } \\
n=22 \text { Children with ADS; } \\
M_{\text {age }}=4.93, S D=2.03, \\
\text { Female }=18.2 \% \text { ) and } \\
20 \text { Caregivers } \\
\text { (Female }=90.0 \% \text { ) of } \\
\text { Children with ASD (control } \\
\text { group, } n=20 \text { Children with } \\
\text { ASD, } M_{\text {age }}=5.65, S D=1.74, \\
\text { Female }=15 \% \text { ) }\end{array}$ & Direct & Parent Education Program & Clinics & 8 Weeks & $\begin{array}{l}\text { Caregiver Burden } \\
\text { Index (CBI) }\end{array}$ & $\begin{array}{l}\text { Independent-Samples } t \text {-test } \\
\text { Parents' mental HRQOL } \\
\text { significantly improved after the } \\
\text { intervention } \\
(t=-2.138 ; p=0.039) \text {. Parents' } \\
\text { physical HRQOL did not improve } \\
\text { after the intervention }(t=-1463 ; \\
\quad p=0.151) .\end{array}$ \\
\hline
\end{tabular}


Table 1. Cont.

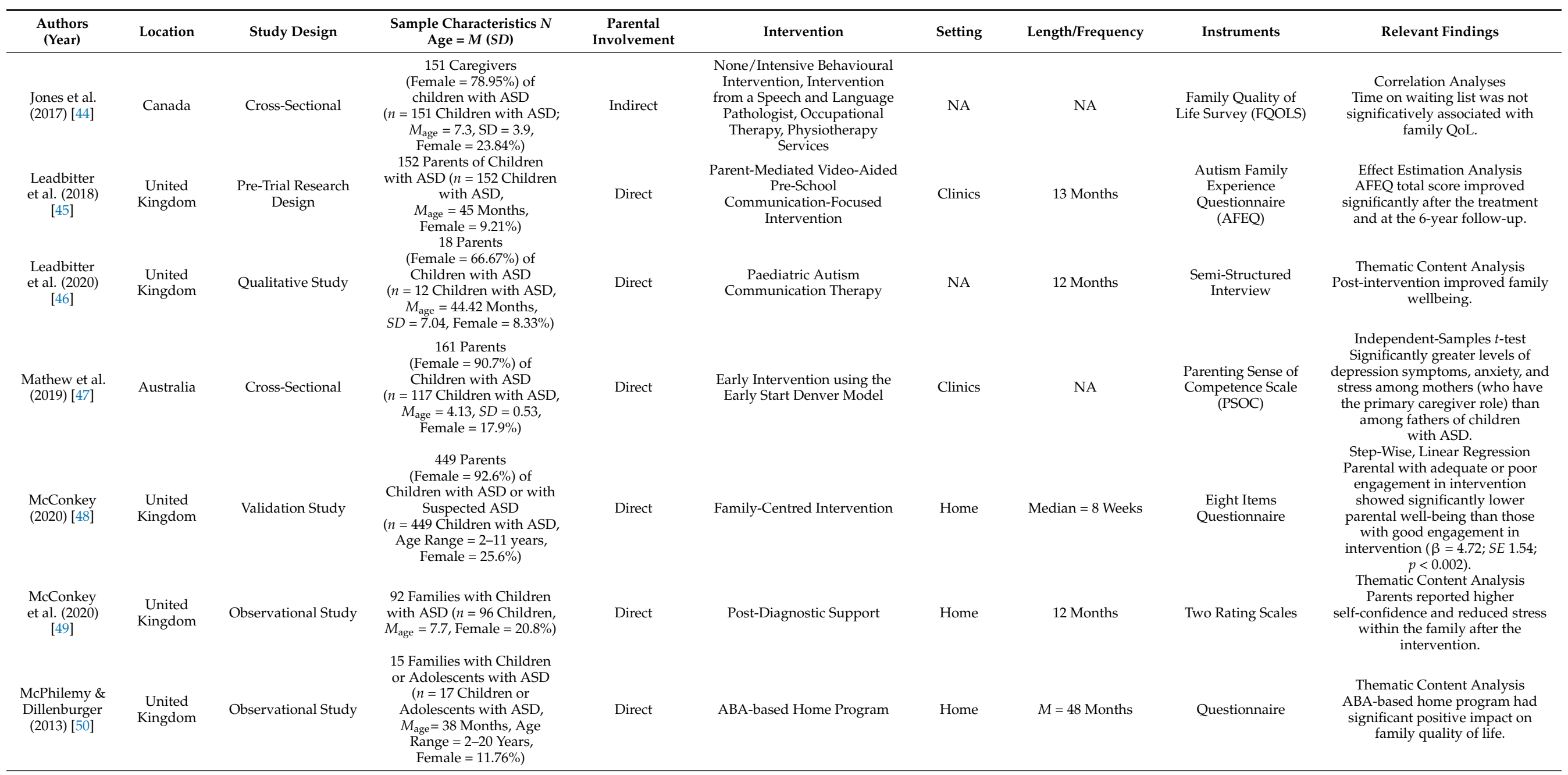


Table 1. Cont

\begin{tabular}{|c|c|c|c|c|c|c|c|c|c|}
\hline $\begin{array}{l}\text { Authors } \\
\text { (Year) }\end{array}$ & Location & Study Design & $\begin{array}{c}\text { Sample Characteristics } N \\
\text { Age }=M(S D)\end{array}$ & $\begin{array}{c}\text { Parental } \\
\text { Involvement }\end{array}$ & Intervention & Setting & Length/Frequency & Instruments & Relevant Findings \\
\hline $\begin{array}{c}\text { Milbourn } \\
\text { et al. (2017) } \\
\text { [51] }\end{array}$ & $\begin{array}{l}\text { Australia/ } \\
\text { Sweden }\end{array}$ & $\begin{array}{l}\text { Observational/ } \\
\text { Qualitative Study }\end{array}$ & $\begin{array}{c}521 \text { Caregivers } \\
(\text { Female }=81 \%) \text { with } \\
\text { Children with ASD } \\
(n=400 \text { Children with ASD } \\
(76.78 \%), M_{\text {age }}=9.92 \\
S D=4.17, \text { Female }=17 \%)\end{array}$ & Direct & Mixed & NA & NA & $\begin{array}{l}\text { Multidimensional } \\
\text { Questionnaire }\end{array}$ & $\begin{array}{l}\text { Thematic Content Analysis } \\
\text { Respondents indicated that early } \\
\text { interventions improved their } \\
\text { family life ( } 71.3 \% \text { answered } \\
\text { "somewhet" or "definitely"). } \\
\text { Respondents agreed that earlier } \\
\text { access to intervention would have } \\
\text { led to improved child's quality of } \\
\text { life ( } 78.4 \% \text { answered "somewhat" } \\
\text { or "definitely"). }\end{array}$ \\
\hline $\begin{array}{l}\text { Moody et al. } \\
\text { (2019) [52] }\end{array}$ & $\begin{array}{l}\text { United } \\
\text { States }\end{array}$ & $\begin{array}{l}\text { Randomised } \\
\text { Controlled Trial }\end{array}$ & $\begin{array}{c}67 \text { Parents (Active Group, } \\
\text { Female }=91.0 \% ; \text { Waitlist } \\
\text { Female }=88.2 \% \text { ) of } \\
\text { Children with ASD } \\
(n=33 \text { Intervention Group, } \\
\text { Female }=18.2 \%) \\
(n=67 \text { Children with ASD, } \\
\text { Range age } 2-8 \text { Years, } \\
\text { Female }=14.7 \%)\end{array}$ & Direct & $\begin{array}{l}\text { Colorado Parent Mentoring } \\
\text { (CPM) Program }\end{array}$ & Mixed & Six Months & $\begin{array}{l}\text { Family Quality of } \\
\text { Life Survey (FQOLS) }\end{array}$ & $\begin{array}{l}\text { Mixed Modelling } \\
\text { CPM program positively impacted } \\
\text { several areas of family quality of } \\
\text { life, regardless the amount of } \\
\text { formal intervention received. }\end{array}$ \\
\hline $\begin{array}{l}\text { Paynter et al. } \\
\text { (2018) [53] }\end{array}$ & Australia & Mixed Methods & $\begin{array}{l}\text { 26 Fathers of Children with } \\
\text { ASD }(n=26 \text { Children with } \\
\text { ASD, Age Range } \\
2.5-6 \text { Years, } \\
\text { Female }=26.4 \%)\end{array}$ & Indirect & Early Intervention & Clinics & NA & $\begin{array}{l}\text { Parenting Stress } \\
\text { Index (PSI) }\end{array}$ & $\begin{array}{l}\text { Thematic Content Analysis } \\
\text { According to some fathers' family } \\
\text { adaptation was negatively affected } \\
\text { by inaccessible and/or } \\
\text { gender-biased formal supports. } \\
\text { Analysis of Covariance }\end{array}$ \\
\hline
\end{tabular}


Table 2. Measurement instruments and construct dimensions listed in the included studies $(n=17)$.

\begin{tabular}{|c|c|c|c|c|}
\hline & Measures of QoL & Construct Dimensions & Studies & $\begin{array}{l}\text { No. of } \\
\text { Studies }\end{array}$ \\
\hline \multirow[t]{10}{*}{ Family Quality of Life } & Caregiver Burden Index (CBI) [55] & $\begin{array}{l}\text { Time Burden, Burden of Personal } \\
\text { Development Limitations, Physical Burden, } \\
\text { Social Burden, Emotional Burden }\end{array}$ & Ji et al. (2014) [43] & 1 \\
\hline & & Emotional Wellbeing, Family Wellbeing, & Hwang et al. (2015) [42]; & \\
\hline & Family Quality of Life (FQOL) [37] & Parenting Wellbeing, Physical/Material & Moody et al. (2019) [52]; & 3 \\
\hline & & Wellbeing, Disability-Related Wellbeing & Roberts et al. (2011) [54] & \\
\hline & $\begin{array}{l}\text { Family Quality of Life Survey } \\
\text { (FQOLS) [56] }\end{array}$ & $\begin{array}{l}\text { About Your Family, Health of the Family, } \\
\text { Support from Disability-Related Services, } \\
\text { Leisure and Recreation, Community } \\
\text { Interaction, Overall Family Quality of Life }\end{array}$ & Jones et al. (2017) [44] & 1 \\
\hline & $\begin{array}{c}\text { Par-DD-QoL } \\
\text { (from Par-ENT-QoL) [57] }\end{array}$ & $\begin{array}{c}\text { Emotional Score, Daily Disturbances Score, } \\
\text { Global Score }\end{array}$ & Baghdadli et al. (2014) [10] & 1 \\
\hline & $\begin{array}{l}\text { Parenting Sense of Competence } \\
\text { Scale (PSOC) [58] }\end{array}$ & Satisfaction, Interest, Efficacy & Mathew et al. (2019) [47] & 1 \\
\hline & Parenting Stress Index (PSI) [38] & $\begin{array}{l}\text { Parental Distress, Parent-Child } \\
\text { Dysfunctional Interaction, Difficult Child }\end{array}$ & $\begin{array}{l}\text { Paynter et al. (2018) [53]; } \\
\text { Roberts et al. (2011) [54] }\end{array}$ & 2 \\
\hline & WHOQOL-BREF [38] & $\begin{array}{l}\text { Four Domains (Physical, Psychological, } \\
\text { Social Relationships, Environment) }\end{array}$ & Derguy et al. (2018) [40] & 1 \\
\hline & $\begin{array}{l}\text { Quality of Life in Autism } \\
\text { questionnaire (QoLA) [59] }\end{array}$ & $\begin{array}{l}\text { Subscale A (Quality of Life), Subscale B } \\
\text { (Impact of ASD Symptoms) }\end{array}$ & Due et al. (2018) [41] & 1 \\
\hline
\end{tabular}

\subsection{Quality Assessment}

We implemented the AXIS tool [34] to evaluate each of the 17 included papers. The average quality score was 13.18 out of a total of 20 points $(\min =7, \max =19, S D=3.68)$, indicating a moderate quality among studies, with two low, eight moderate, and seven high quality ratings (Table 3). Globally, the aims and the objectives were clearly indicated (question number one) and the design was appropriate for the stated aims (question number two). Only three papers $[40,43,54]$, however, reported a specific calculation of the effect size (question number three) to justify the chosen sample. Since the selected interventions were specifically aimed at children with ASD and their parents, the target population was clearly defined in 12 studies (question number four), and the selection process was found to be adequate for most of the studies (questions number five and six). Non-responders were infrequent in the reviewed samples, nevertheless, only one study [43] explicitly reported the measures undertaken to address them (question number seven). The outcome variables were appropriately estimated with suitable instruments in approximately half of the included studies (question number eight, negative scores $n=7$; question number nine, negative scores $n=7$ ), and the statistical methods and data were globally specified in sufficient detail (questions from 10 to 12). Overall, the discussions and authors' conclusions were justified by the achieved results, and the inherent limitations of the study designs were correctly presented (question number 18, negative scores $n=4$ ). 
Table 3. Quality assessments and total scores using the Appraisal Tool for Cross-Sectional Studies (AXIS).

\begin{tabular}{|c|c|c|c|c|c|c|c|c|c|c|c|c|c|c|c|c|c|c|c|c|c|c|}
\hline Author (Year) & Q1 & Q2 & Q3 & Q4 & Q5 & Q6 & Q7 & Q8 & Q9 & Q10 & Q11 & Q12 & Q13* & Q14 & Q15 & Q16 & Q17 & Q18 & Q19* & Q20 & $\begin{array}{l}\text { Quality } \\
\text { Score/20 }\end{array}$ & $\begin{array}{l}\text { Quality } \\
\text { Rating }\end{array}$ \\
\hline $\begin{array}{l}\text { Aandersson et al. } \\
\text { (2017) [39] }\end{array}$ & $\mathrm{Y}$ & $\mathrm{Y}$ & $\mathrm{N}$ & $\mathrm{N}$ & $\mathrm{Y}$ & $\mathrm{N}$ & $\mathrm{N}$ & $\mathrm{N}$ & $\mathrm{N}$ & $\mathrm{N}$ & $\mathrm{N}$ & $\mathrm{Y}$ & $\mathrm{N}$ & $\mathrm{N}$ & $\mathrm{Y}$ & $\mathrm{Y}$ & $\mathrm{Y}$ & Y & $\mathrm{N}$ & $\mathrm{Y}$ & 11 & Moderate \\
\hline $\begin{array}{l}\text { Baghdadli et al. } \\
\text { (2014) [10] }\end{array}$ & Y & $\mathrm{N}$ & $\mathrm{N}$ & Y & $\mathrm{Y}$ & $\mathrm{Y}$ & $\mathrm{N}$ & $\mathrm{N}$ & $\mathrm{N}$ & $\mathrm{Y}$ & $\mathrm{N}$ & $\mathrm{Y}$ & $\mathrm{N}$ & $\mathrm{N}$ & $\mathrm{Y}$ & $\mathrm{Y}$ & $\mathrm{Y}$ & $\mathrm{Y}$ & $\mathrm{N}$ & Y & 13 & Moderate \\
\hline $\begin{array}{l}\text { Derguy et al. } \\
\text { (2018) [40] }\end{array}$ & Y & $\mathrm{Y}$ & $\mathrm{Y}$ & $\mathrm{Y}$ & $\mathrm{Y}$ & $\mathrm{Y}$ & $\mathrm{N}$ & $\mathrm{Y}$ & $\mathrm{Y}$ & $\mathrm{Y}$ & $\mathrm{N}$ & $\mathrm{Y}$ & $\mathrm{N}$ & $\mathrm{N}$ & $\mathrm{Y}$ & $\mathrm{Y}$ & $\mathrm{Y}$ & $\mathrm{Y}$ & $\mathrm{N}$ & $\mathrm{Y}$ & 17 & High \\
\hline $\begin{array}{l}\text { Due et al. } \\
\text { (2018) [41] }\end{array}$ & Y & $\mathrm{Y}$ & $\mathrm{N}$ & $\mathrm{N}$ & $\mathrm{Y}$ & $\mathrm{N}$ & $\mathrm{N}$ & $\mathrm{N}$ & $\mathrm{N}$ & $\mathrm{N}$ & $\mathrm{N}$ & $\mathrm{N}$ & $\mathrm{N}$ & $\mathrm{N}$ & $\mathrm{N}$ & $\mathrm{Y}$ & $\mathrm{Y}$ & $\mathrm{Y}$ & $\mathrm{N}$ & $\mathrm{Y}$ & 10 & Moderate \\
\hline $\begin{array}{l}\text { Hwang et al. } \\
\text { (2015) [42] }\end{array}$ & Y & Y & $\mathrm{N}$ & Y & Y & $\mathrm{N}$ & $\mathrm{N}$ & Y & Y & $\mathrm{N}$ & $\mathrm{Y}$ & $\mathrm{Y}$ & $\mathrm{N}$ & $\mathrm{N}$ & Y & Y & Y & Y & na & Y & 14 & Moderate \\
\hline $\begin{array}{l}\text { Ji et al. } \\
\text { (2014) [43] }\end{array}$ & $\mathrm{N}$ & $\mathrm{Y}$ & $\mathrm{Y}$ & $\mathrm{Y}$ & $\mathrm{Y}$ & $\mathrm{Y}$ & $\mathrm{Y}$ & $\mathrm{Y}$ & $\mathrm{Y}$ & $\mathrm{Y}$ & $\mathrm{Y}$ & $\mathrm{Y}$ & $\mathrm{N}$ & $\mathrm{Y}$ & $\mathrm{Y}$ & Y & Y & $\mathrm{Y}$ & $\mathrm{N}$ & Y & 19 & High \\
\hline $\begin{array}{l}\text { Jones et al. } \\
\text { (2017) [44] }\end{array}$ & Y & $\mathrm{Y}$ & $\mathrm{N}$ & Y & $\mathrm{Y}$ & Y & $\mathrm{N}$ & Y & $\mathrm{Y}$ & $\mathrm{N}$ & $\mathrm{N}$ & $\mathrm{Y}$ & $\mathrm{N}$ & $\mathrm{N}$ & $\mathrm{Y}$ & Y & $\mathrm{Y}$ & $\mathrm{Y}$ & $\mathrm{N}$ & $\mathrm{Y}$ & 15 & High \\
\hline $\begin{array}{l}\text { Leadbitter et al. } \\
\quad(2018) \text { [45] }\end{array}$ & Y & Y & $\mathrm{N}$ & $\mathrm{N}$ & Y & $\mathrm{N}$ & $\mathrm{N}$ & $\mathrm{N}$ & Y & $\mathrm{N}$ & $\mathrm{N}$ & Y & $\mathrm{N}$ & $\mathrm{N}$ & Y & Y & $\mathrm{Y}$ & $\mathrm{N}$ & $\mathrm{N}$ & Y & 11 & Moderate \\
\hline $\begin{array}{l}\text { Leadbitter et al. } \\
\quad(2020)[46]\end{array}$ & Y & $\mathrm{Y}$ & $\mathrm{N}$ & Y & $\mathrm{Y}$ & $\mathrm{Y}$ & $\mathrm{N}$ & $\mathrm{Y}$ & $\mathrm{N}$ & $\mathrm{N}$ & $\mathrm{N}$ & Y & $\mathrm{N}$ & $\mathrm{N}$ & Y & Y & $\mathrm{Y}$ & Y & $\mathrm{N}$ & Y & 14 & Moderate \\
\hline $\begin{array}{l}\text { Mathew et al. } \\
\text { (2019) [47] }\end{array}$ & Y & $\mathrm{Y}$ & $\mathrm{N}$ & Y & $\mathrm{Y}$ & Y & $\mathrm{N}$ & $\mathrm{Y}$ & Y & Y & $\mathrm{N}$ & Y & $\mathrm{N}$ & $\mathrm{N}$ & $\mathrm{Y}$ & Y & Y & Y & $\mathrm{N}$ & Y & 16 & High \\
\hline $\begin{array}{l}\text { McConkey et al. } \\
(2020) \text { [49] } \\
\text { McPhilemy \& }\end{array}$ & $\mathrm{N}$ & $\mathrm{Y}$ & $\mathrm{N}$ & Y & $\mathrm{N}$ & $\mathrm{N}$ & $\mathrm{N}$ & $\mathrm{N}$ & $\mathrm{N}$ & $\mathrm{N}$ & $\mathrm{N}$ & Y & $\mathrm{N}$ & $\mathrm{N}$ & Y & $\mathrm{N}$ & $\mathrm{N}$ & $\mathrm{N}$ & $\mathrm{N}$ & Y & 7 & Low \\
\hline $\begin{array}{l}\text { Dillenburger } \\
\text { (2013) [50] }\end{array}$ & $\mathrm{N}$ & $\mathrm{N}$ & $\mathrm{N}$ & $\mathrm{N}$ & $\mathrm{Y}$ & $\mathrm{N}$ & $\mathrm{N}$ & $\mathrm{N}$ & $\mathrm{N}$ & $\mathrm{N}$ & $\mathrm{N}$ & $\mathrm{Y}$ & $\mathrm{N}$ & $\mathrm{N}$ & Y & $\mathrm{Y}$ & $\mathrm{Y}$ & $\mathrm{N}$ & na & $\mathrm{Y}$ & 7 & Low \\
\hline $\begin{array}{l}\text { Milbourn et al. } \\
\text { (2017) [51] }\end{array}$ & Y & $\mathrm{Y}$ & $\mathrm{N}$ & Y & $\mathrm{N}$ & $\mathrm{N}$ & $\mathrm{N}$ & na & na & $\mathrm{N}$ & $\mathrm{N}$ & $\mathrm{N}$ & $\mathrm{N}$ & $\mathrm{N}$ & $\mathrm{Y}$ & Y & Y & Y & $\mathrm{N}$ & Y & 10 & Moderate \\
\hline $\begin{array}{l}\text { Moody et al. } \\
\text { (2019) [52] }\end{array}$ & Y & Y & $\mathrm{N}$ & Y & Y & $\mathrm{Y}$ & $\mathrm{N}$ & Y & Y & $\mathrm{Y}$ & Y & Y & $\mathrm{N}$ & $\mathrm{N}$ & $\mathrm{Y}$ & Y & Y & $\mathrm{Y}$ & $\mathrm{N}$ & Y & 17 & High \\
\hline $\begin{array}{l}\text { Paynter et al. } \\
\text { (2018) [53] }\end{array}$ & $\mathrm{Y}$ & $\mathrm{Y}$ & $\mathrm{N}$ & Y & $\mathrm{Y}$ & Y & na & Y & Y & $\mathrm{N}$ & Y & $\mathrm{Y}$ & $\mathrm{N}$ & $\mathrm{N}$ & $\mathrm{Y}$ & Y & Y & $\mathrm{Y}$ & $\mathrm{N}$ & Y & 16 & High \\
\hline $\begin{array}{l}\text { Roberts et al. } \\
\text { (2011) [54] }\end{array}$ & Y & $\mathrm{Y}$ & $\mathrm{Y}$ & Y & $\mathrm{Y}$ & Y & $\mathrm{N}$ & Y & $\mathrm{Y}$ & Y & Y & Y & $\mathrm{N}$ & $\mathrm{N}$ & Y & Y & Y & Y & $\mathrm{N}$ & Y & 18 & High \\
\hline $\begin{array}{c}\text { Mean } \\
\text { Standard Deviation }\end{array}$ & & & & & & & & & & & & & & & & & & & & & $\begin{array}{c}13.18 \\
3.68\end{array}$ & \\
\hline
\end{tabular}




\subsection{Main Findings}

\subsubsection{No Parental Involvement}

In two studies, interventions were conducted entirely by professionals without any form of parental involvement [10,40]. Derguy et al. [40] found that access to child-focused psycho-educational intervention was positively associated with parental QoL. Baghdadli et al. [10] found that the number of hours of specialized intervention focused on externalizing difficulties of adolescents with ASD was negatively associated with parental QoL.

\subsubsection{Indirect Parental Involvement}

Four studies used indirect strategies (e.g., parent training and support groups) to involve parents in the interventions $[39,44,53,54]$. In these cases, the main part of the intervention is centred on the child or the adolescent with ASD and is conducted in a clinical setting.

The results indicated an overall low level of QoL among parents who have been indirectly involved in an intervention for their child or adolescent with ASD. Specifically, Paynter et al. [53] found low levels of family adaptation (e.g., individual mental health, relationship quality, and family well-being) among fathers of children with ASD who participated to the centre-based early intervention. Interventions based on applied behaviour analysis (ABA) were associated with overall negative parent experiences because of the poor quality of the support received at home and the scarce coordination and guidance from the professionals [39]. In addition, findings by Jones et al. [44] showed that time on a waitlist for the ABA treatment was not associated with parental QoL. In contrast, Roberts et al.'s [54] randomised control trial revealed an increase in parent QoL after centrebased early interventions focused on functional impairments of children and on improving the parents' knowledge of ASD.

\subsubsection{Direct Parental Involvement}

Twelve studies used direct methods (e.g., parent-mediated home-based training, family-centred intervention, parent mentoring program) to involve parents in the interventions [41-43,45,47-52].

The majority of studies found that parental QoL improved after the intervention and, crucially, two studies found that parental direct involvement in the intervention was associated with greater parental QoL [41,43]. Specifically, parents of children with ASD showed significantly improved QoL after they participated in a parent-mediated communicationfocused intervention [45,46], a family-centred intervention [49], an ABA-based home program [50], and the Colorado parent mentoring (CPM) program [52]. Moreover, parents of children with ASD who participated in a multidisciplinary parent education program reported increased mental, but not physical, QoL [43,48]. Consistently, Milbourn et al. [51] found that parents of children and adolescents with ASD reported that early access to intervention improved their QoL, especially in terms of fostering their involvement in their child's daily life. However, three studies reported inconsistent results. Roberts et al. [48] found no significant changes in the QoL of parents of children with ASD after a home-based early intervention program. Similarly, Hwang et al. [42] found that mothers of children and adolescents with ASD did not report a significant increase in their QoL after they received a mindfulness parent-mediated home-based training. In addition, parents, especially mothers of children with ASD, who received an early intervention using the Early Start Denver Model showed lower parental well-being compared to the normative population [47].

\section{Discussion}

To our knowledge, this is the first review to examine the relationship between QoL and involvement in intervention among parents of children and adolescents with ASD. The 17 reviewed studies involved a total of 18 interventions targeting children and adolescents with ASD. Two studies reported no parental involvement in the intervention, three studies reported indirect parental involvement, and 11 studies reported direct parental 
involvement. One study [54] included both direct and indirect parental involvement in intervention.

The reviewed studies used heterogeneous designs and data collection methods and the majority of the studies had relatively small sample sizes $[39,41-43,46,49,50,52-54]$, which limits our ability to draw firm conclusions about the association between parental QoL and involvement in interventions for youth with ASD.

From the limited data of the reviewed studies, the overall QoL of parents of children and adolescents with ASD appears to be shaped by the way they have been involved in the intervention. In line with a previous systematic review [24], our results showed that access to ASD services is a relevant protective factor not only for children, but also for parents [40]. Children with ASD can be expected to gain significant improvements when diagnosed early and when engaged in structured, intensive, evidence-based programs [61-63]. In contrast, difficulties in meeting the service needs of children with ASDs, ranging from general medical services to supportive services [64], can generate a vicious cycle of worsening symptoms and an overload of responsibility on parents who come to feel exhausted and ineffective [39]. However, the fact that one's child with ASD receives treatment does not in itself guarantee an improvement in parental QoL. In fact, results showed that interventions based on the external difficulties of people with ASD, which do not involve parents in the intervention or involve them only indirectly, do not improve or even worsen parental QoL [10,39,44]. For example, interventions based on ABA are recognised as valid methods used for educating individuals with ASD [65,66], which are characterised by a strong asymmetry of roles of participants in the intervention. Qualified behaviour analysts design, develop, conduct, and directly oversee the ABA-programme that is focused on the child or the adolescent with ASD, while parents receive training to support their offspring in skill practice throughout the day. Behavioural child-focused interventions (e.g., ABA-based interventions), no matter how clinically effective, may be not sufficiently adapted to the broad family needs or may be unsuitable for a family situation. In contrast, when ABA-based interventions are implemented in a home setting and actively involved the whole family, parents report an overall better QoL [50]. In fact, interventions explicitly targeting children's daily living skills in addition to the hallmark symptoms of ASD (i.e., social-communication deficits and repetitive, restrictive behaviours) may enhance parental QoL [54,67].

The majority of the studies examined in this review directly involved parents in the intervention. This may be a sign in the shift in perspective that is occurring in interventions for individuals with ASD and an increased awareness by professionals of the role of parents to implement effective interventions [46]. Parents who were directly involved in the intervention tended to report a higher QoL [43,45,46,49-52]. For example, in one study [43], 22 caregivers of children with ASD participated in an 8-week multidisciplinary education programme, with the intent to learn adaptive strategies and manage the problem behaviours of their child, and consequently to improve the intrafamilial QoL. Results indicated a significant improvement in family functioning, self-efficacy, and coping styles. Another study [49] provided post-diagnostic support to nearly 100 families and children, over a 12-month period. The project implemented a family-centred plan that showed improvements in children's social and communication skills and overall stress reduction in parents. Yet another study [42] described an 8-week training program that comprised six mother-child dyads, aimed at teaching mindfulness strategies to better cope with problem behaviours of their children. At the end of the program, the participants reported positive effects in the targeted areas including parenting stress, intrafamilial QoL, and problematic behaviours. These results can be attributable to various reasons. When parents are actively involved by professionals, the intervention is more personalised and ecological [40]. This is also a way of empowering parents by recognising their expertise and contributions $[68,69]$ and to develop constructive collaboration between different actors involved in the intervention [70]. Based on these findings, clinicians should assess and counsel parents, and identify potential barriers (e.g., lack of resolution of diagnosis) 
that may prevent them from actively participating in the intervention for their child or adolescent with ASD.

\section{Limitations}

The present systematic review comes with a number of limitations. Some depend on the relative novelty of the topic and on the absence of shared guidelines in the treatment of children and adolescents with ASD. The selected studies adopted a variety of methods and approaches (e.g., observational studies vs. randomised controlled trials), which limit the possibility to compare their findings. Another limitation regards our search and selection procedure. The construct of parental QoL is very broad and thus some studies may have evaluated some specific dimensions not included in our search keywords. Additionally, we only included articles published in English sources, which may have contributed to a selection bias by overlooking relevant articles published in other languages. In addition, we did not investigate the QoL of family as a whole (e.g., including siblings of children with ASD). However, parents are more generally actively involved in the care and intervention for their children. Furthermore, geographical coverage was dominated by Europe and Australia, therefore further research is needed in Asiatic cultural contexts [71]. Finally, the included studies recruited relatively small samples of self-selected participants who may not be necessarily representative of the general population.

\section{Conclusions and Future Directions}

Parental involvement in intervention for children or adolescents with ASD is a topic that is attracting increasing attention, as evidenced by the fact that parents were directly involved in interventions in most of the studies selected in this systematic review. The findings suggest that increasing parental involvement in the intervention for children or adolescents with ASD may be one way to promote their QoL. More precisely, this systematic review highlights that a constructive collaboration between professionals and parents in planning and executing interventions may promote more ecological results and better satisfaction among parents. This finding is consistent with the literature on parental QoL [24] and supports the relevance of an ecological and holistic approach in the research on QoL in parents of children with ASD [40]. This could help clinicians to better identify and address parental needs and enhance parenting resources that have a positive impact on the intervention.

Suggestions for future research put forward here include the use of longitudinal studies conducted with wider and demographically diverse samples that would include families from diverse geographical locations who have children with ASD of different ages (e.g., adults).

Supplementary Materials: The following are available online at https:/ / www.mdpi.com/article/10 $.3390 / j p m 11090894 /$ s1, Table S1: Full-text articles excluded, with reasons $(n=79)$.

Author Contributions: Conceptualization, A.M. and T.M.; Methodology, A.M. and T.M.; Writingoriginal draft preparation, A.M. and T.M.; Writing—review and editing, B.D., C.R., G.B., R.M., G.E. and D.D.; Supervision, G.P., C.F. and P.C. All authors have read and agreed to the published version of the manuscript.

Funding: The APC was funded by Fondazione Bambini e Autismo Onlus.

Institutional Review Board Statement: Not applicable.

Informed Consent Statement: Informed consent was obtained from all subjects involved in the study.

Data Availability Statement: Not applicable.

Conflicts of Interest: The authors declare no conflict of interest. 


\section{References}

1. American Psychiatric Association. Diagnostic and Statistical Manual of Mental Disorders, 5th ed.; American Psychiatric Association: Washington, DC, USA, 2013; ISBN 0-89042-555-8.

2. Volkmar, F.R.; Pauls, D. Autism. Lancet 2003, 362, 1133-1141. [CrossRef]

3. Giovagnoli, G.; Postorino, V.; Fatta, L.M.; Sanges, V.; De Peppo, L.; Vassena, L.; De Rose, P.; Vicari, S.; Mazzone, L. Behavioral and emotional profile and parental stress in preschool children with autism spectrum disorder. Res. Dev. Disabil. 2015, 45-46, 411-421. [CrossRef]

4. Militerni, R.; Bravaccio, C.; Falco, C.; Fico, C.; Palermo, M.T. Repetitive behaviors in autistic disorder. Eur. Child Adolesc. Psychiatry 2002, 11, 210-218. [CrossRef] [PubMed]

5. Tudor, M.E.; Hoffman, C.D.; Sweeney, D.P. Children with autism: Sleep problems and symptom severity. Focus Autism Other Dev. Disabl. 2012, 27, 254-262. [CrossRef]

6. Shaw, A.; Do, T.N.T.; Harrison, L.; Marczak, M.; Dimitriou, D.; Joyce, A. Sleep and cognition in people with autism spectrum condition: A systematic literature review. Rev. J. Autism Dev. Disord. 2021. [CrossRef]

7. Pozo, P.; Sarriá, E.; Brioso, A. Family quality of life and psychological well-being in parents of children with autism spectrum disorders: A double ABCX model. J. Intellect. Disabil. Res. 2014, 58, 442-458. [CrossRef]

8. Corsano, P.; Musetti, A.; Guidotti, L.; Capelli, F. Typically developing adolescents' experience of growing up with a brother with an autism spectrum disorder. J. Intellect. Dev. Disabil. 2017, 42, 151-161. [CrossRef]

9. Guidotti, L.; Musetti, A.; Barbieri, G.L.; Ballocchi, I.; Corsano, P. Conflicting and harmonious sibling relationships of children and adolescent siblings of children with autism spectrum disorder. Child Care Health Dev. 2021, 47, 163-173. [CrossRef] [PubMed]

10. Baghdadli, A.; Pry, R.; Michelon, C.; Rattaz, C. Impact of autism in adolescents on parental quality of life. Qual. Life Res. 2014, 23, 1859-1868. [CrossRef] [PubMed]

11. Hayes, S.A.; Watson, S.L. The impact of parenting stress: A meta-analysis of studies comparing the experience of parenting stress in parents of children with and without autism spectrum disorder. J. Autism Dev. Disord. 2013, 43, 629-642. [CrossRef] [PubMed]

12. Bonis, S. Stress and parents of children with autism: A review of literature. Issues Ment. Health Nurs. 2016, 37, 153-163. [CrossRef] [PubMed]

13. Davis, N.O.; Carter, A.S. Parenting stress in mothers and fathers of toddlers with autism spectrum disorders: Associations with child characteristics. J. Autism Dev. Disord. 2008, 38, 1278-1291. [CrossRef]

14. Factor, R.S.; Ollendick, T.H.; Cooper, L.D.; Dunsmore, J.C.; Rea, H.M.; Scarpa, A. All in the family: A systematic review of the effect of caregiver-administered autism spectrum disorder interventions on family functioning and relationships. Clin. Child Fam. Psychol. Rev. 2019, 22, 433-457. [CrossRef] [PubMed]

15. McConachie, H.; Diggle, T. Parent implemented early intervention for young children with autism spectrum disorder: A systematic review. J. Eval. Clin. Pract. 2007, 13, 120-129. [CrossRef]

16. Yan, T.; Hou, Y.; Deng, M. Direct, indirect, and buffering effect of social support on parental involvement among Chinese parents of children with autism spectrum disorders. J. Autism Dev. Disord. 2021. [CrossRef]

17. Đorđević, M.; Glumbić, N.; Memisevic, H.; Brojčin, B.; Krstov, A. Parent-teacher interactions, family stress, well-being, and parental depression as contributing factors to parental involvement mechanisms in education of children with autism. Int. J. Dev. Disabil. 2021, 1-12. [CrossRef]

18. Schertz, H.H.; Baker, C.; Hurwitz, S.; Benner, L. Principles of early intervention reflected in toddler research in autism spectrum disorders. Top. Early Child. Spec. Educ. 2011, 31, 4-21. [CrossRef]

19. Liu, Q.; Hsieh, W.Y.; Chen, G. A systematic review and meta-analysis of parent-mediated intervention for children and adolescents with autism spectrum disorder in mainland China, Hong Kong, and Taiwan. Autism 2020, 24, 1960-1979. [CrossRef]

20. Karst, J.S.; van Hecke, A.V. Parent and family impact of autism spectrum disorders: A review and proposed model for intervention evaluation. Clin. Child Fam. Psychol. Rev. 2012, 15, 247-277. [CrossRef]

21. Payakachat, N.; Tilford, J.M.; Kovacs, E.; Kuhlthau, K. Autism spectrum disorders: A review of measures for clinical, health services and cost-effectiveness applications. Expert Rev. Pharmacoecon. Outcomes Res. 2012, 12, 485-503. [CrossRef]

22. Hastings, R.P.; Taunt, H.M. Positive perceptions in families of children with developmental disabilities. Am. J. Ment. Retard. 2002, 107, 116. [CrossRef]

23. Cappe, E.; Wolff, M.; Bobet, R.; Adrien, J.L. Quality of life: A key variable to consider in the evaluation of adjustment in parents of children with autism spectrum disorders and in the development of relevant support and assistance programmes. Qual. Life Res. 2011, 20, 1279-1294. [CrossRef] [PubMed]

24. Vasilopoulou, E.; Nisbet, J. The quality of life of parents of children with autism spectrum disorder: A systematic review. Res. Autism Spectr. Disord. 2016, 23, 36-49. [CrossRef]

25. Hastings, R.P.; Kovshoff, H.; Ward, N.J.; Degli Espinosa, F.; Brown, T.; Remington, B. Systems analysis of stress and positive perceptions in mothers and fathers of pre-school children with autism. J. Autism Dev. Disord. 2005, 35, 635-644. [CrossRef] [PubMed]

26. Lickenbrock, D.M.; Ekas, N.V.; Whitman, T.L. Feeling good, feeling bad: Influences of maternal perceptions of the child and marital adjustment on well-being in mothers of children with an autism spectrum disorder. J. Autism Dev. Disord. 2011, 41, 848-858. [CrossRef] 
27. Wachtel, K.; Carter, A.S. Reaction to diagnosis and parenting styles among mothers of young children with ASDs. Autism 2008, 12, 575-594. [CrossRef]

28. Dardas, L.A.; Ahmad, M.M. Validation of the World Health Organization's quality of life questionnaire with parents of children with autistic disorder. J. Autism Dev. Disord. 2014, 44, 2257-2263. [CrossRef] [PubMed]

29. Mello, C.; Rivard, M.; Terroux, A.; Mercier, C. Quality of life in families of young children with autism spectrum disorder. Am. J. Intellect. Dev. Disabil. 2019, 124, 535-548. [CrossRef]

30. WHO Quality of Life Assessment Group. The World Health Organization quality of life assessment (WHOQOL): Development and general psychometric properties. Soc. Sci. Med. 1998, 46, 1569-1585. [CrossRef]

31. Dixon, L.; Lucksted, A.; Stewart, B.; Burland, J.; Brown, C.H.; Postrado, L.; McGuire, C.; Hoffman, M. Outcomes of the peer-taught 12-week family-to-family education program for severe mental illness. Acta Psychiatr. Scand. 2004, 109, 207-215. [CrossRef]

32. Liberati, A.; Altman, D.G.; Tetzlaff, J.; Mulrow, C.; Gøtzsche, P.C.; Ioannidis, J.P.A.; Clarke, M.; Devereaux, P.J.; Kleijnen, J.; Moher, D. The PRISMA statement for reporting systematic reviews and meta-analyses of studies that evaluate health care interventions: Explanation and elaboration. J. Clin. Epidemiol. 2009, 62, e1-e34. [CrossRef]

33. Edwards, P.; Clarke, M.; DiGuiseppi, C.; Pratap, S.; Roberts, I.; Wentz, R. Identification of randomized controlled trials in systematic reviews: Accuracy and reliability of screening records. Stat. Med. 2002, 21, 1635-1640. [CrossRef] [PubMed]

34. Downes, M.J.; Brennan, M.L.; Williams, H.C.; Dean, R.S. Development of a critical appraisal tool to assess the quality of cross-sectional studies (AXIS). BMJ Open 2016, 6, 1-7. [CrossRef]

35. Moor, L.; Anderson, J.R. A systematic literature review of the relationship between dark personality traits and antisocial online behaviours. Pers. Individ. Dif. 2019, 144, 40-55. [CrossRef]

36. Raber, M.; Swartz, M.C.; Santa Maria, D.; O'Connor, T.; Baranowski, T.; Li, R.; Chandra, J. Parental involvement in exercise and diet interventions for childhood cancer survivors: A systematic review. Pediatr. Res. 2016, 80, 338-346. [CrossRef] [PubMed]

37. Hoffman, L.; Marquis, J.; Poston, D.; Summers, J.A.; Turnbull, A. Assessing family outcomes: Psychometric evaluation of the beach center family quality of life scale. J. Marriage Fam. 2006, 68, 1069-1083. [CrossRef]

38. Abidin, R.R. Parenting Stress Index-Short Form; Psychological Assessment Resources: Lutz, FL, USA, 1995.

39. Aandersson, G.W.; Miniscalco, C.; Ggillberg, N. A 6-year follow-up of children assessed for suspected autism spectrum disorder: Parents' experiences of society's support. Neuropsychiatr. Dis. Treat. 2017, 13, 1783-1796. [CrossRef] [PubMed]

40. Derguy, C.; Roux, S.; Portex, M.; M'bailara, K. An ecological exploration of individual, family, and environmental contributions to parental quality of life in autism. Psychiatry Res. 2018, 268, 87-93. [CrossRef] [PubMed]

41. Due, C.; Goodwin Smith, I.; Allen, P.; Button, E.; Cheek, C.; Quarmby, L.; Stephens, M.; Paku, S.; Ferguson, S.; Fordyce, K. A pilot study of social inclusion and quality of life for parents of children with autism spectrum disorder. J. Intellect. Dev. Disabil. 2018, 43, 73-82. [CrossRef]

42. Hwang, Y.S.; Kearney, P.; Klieve, H.; Lang, W.; Roberts, J. Cultivating mind: Mindfulness interventions for children with autism spectrum disorder and problem behaviours, and their mothers. J. Child Fam. Stud. 2015, 24, 3093-3106. [CrossRef]

43. Ji, B.; Sun, M.; Yi, R.; Tang, S. Multidisciplinary parent education for caregivers of children with autism spectrum disorders. Arch. Psychiatr. Nurs. 2014, 28, 319-326. [CrossRef] [PubMed]

44. Jones, S.; Bremer, E.; Lloyd, M. Autism spectrum disorder: Family quality of life while waiting for intervention services. Qual. Life Res. 2017, 26, 331-342. [CrossRef]

45. Leadbitter, K.; Aldred, C.; McConachie, H.; Le Couteur, A.; Kapadia, D.; Charman, T.; Macdonald, W.; Salomone, E.; Emsley, R.; Green, J.; et al. The Autism Family Experience Questionnaire (AFEQ): An ecologically-valid, parent-nominated measure of family experience, quality of life and prioritised outcomes for early intervention. J. Autism Dev. Disord. 2018, 48, 1052-1062. [CrossRef]

46. Leadbitter, K.; Macdonald, W.; Taylor, C.; Buckle, K.L.; Aldred, C.; Barrett, B.; Barron, S.; Beggs, K.; Blazey, L.; Bourne, K.; et al Parent perceptions of participation in a parent-mediated communication-focussed intervention with their young child with autism spectrum disorder. Autism 2020, 24, 2129-2141. [CrossRef]

47. Mathew, N.E.; Burton, K.L.O.; Schierbeek, A.; Črnčec, R.; Walter, A.; Eapen, V. Parenting preschoolers with autism: Socioeconomic influences on wellbeing and sense of competence. World J. Psychiatry 2019, 9, 30-46. [CrossRef] [PubMed]

48. McConkey, R. A brief measure of parental wellbeing for use in evaluations of family-centred interventions for children with developmental disabilities. Children 2020, 7, 120. [CrossRef]

49. McConkey, R.; Cassin, M.T.; McNaughton, R. Promoting the social inclusion of children with ASD: A family-centred intervention. Brain Sci. 2020, 10, 318. [CrossRef] [PubMed]

50. Mcphilemy, C.; Dillenburger, K. Parents' experiences of applied behaviour analysis (ABA)-based interventions for children diagnosed with autistic spectrum disorder. Br. J. Spec. Educ. 2013, 40, 154-161. [CrossRef]

51. Milbourn, B.; Falkmer, M.; Black, M.H.; Girdler, S.; Falkmer, T.; Horlin, C. An exploration of the experience of parents with children with autism spectrum disorder after diagnosis and intervention. Scand. J. Child Adolesc. Psychiatry Psychol. 2017, 5 , 104-110. [CrossRef]

52. Moody, E.J.; Kaiser, K.; Sharp, D.; Kubicek, L.F.; Rigles, B.; Davis, J.; McSwegin, S.; D'Abreu, L.C.; Rosenberg, C.R. Improving family functioning following diagnosis of ASD: A randomized trial of a parent mentorship program. J. Child Fam. Stud. 2019, 28, 424-435. [CrossRef]

53. Paynter, J.; Davies, M.; Beamish, W. Recognising the "forgotten man": Fathers' experiences in caring for a young child with autism spectrum disorder. J. Intellect. Dev. Disabil. 2017, 43, 112-124. [CrossRef] 
54. Roberts, J.; Williams, K.; Carter, M.; Evans, D.; Parmenter, T.; Silove, N.; Clark, T.; Warren, A. A randomised controlled trial of two early intervention programs for young children with autism: Centre-based with parent program and home-based. Res. Autism Spectr. Disord. 2011, 5, 1553-1566. [CrossRef]

55. Novak, M.; Guest, C. Application of a multidimensional caregiver. Gerontologist 1989, 29, 798-803. [CrossRef] [PubMed]

56. Brown, I.; Brown, R.; Baum, N.; Isaacs, B.; Myerscough, T.; Neikrug, S.; Roth, D.; Shearer, J.; Wang, M. Family Quality of Life Survey: Main Caregivers of People with Intellectual or Developmental Disabilities; Surrey Place Centre: Toronto, ON, Canada, 2006.

57. Berdeaux, G.; Hervié, C.; Smajda, C.; Marquis, P. Parental quality of life and recurrent ENT infections in their children: Development of a questionnaire. Qual. Life Res. 1998, 7, 501-512. [CrossRef] [PubMed]

58. Johnston, C.; Mash, E.J. A measure of parenting satisfaction and efficacy. J. Clin. Child Psychol. 1989, 18, 167-175. [CrossRef]

59. Eapen, V.; Črnčec, R.; Walter, A.; Tay, K.P. Conceptualisation and development of a quality of life measure for parents of children with autism spectrum disorder. Autism Res. Treat. 2014, 2014, 160783. [CrossRef]

60. Harst, L.; Lantzsch, H.; Scheibe, M. Theories predicting end-user acceptance of telemedicine use: Systematic review. J. Med. Internet Res. 2019, 21, e13117. [CrossRef] [PubMed]

61. Lake, J.K.; Tablon Modica, P.; Chan, V.; Weiss, J.A. Considering efficacy and effectiveness trials of cognitive behavioral therapy among youth with autism: A systematic review. Autism 2020, 24, 1590-1606. [CrossRef] [PubMed]

62. Eisenhower, A.; Martinez Pedraza, F.; Sheldrick, R.C.; Frenette, E.; Hoch, N.; Brunt, S.; Carter, A.S. Multi-stage screening in early intervention: A critical strategy for improving ASD identification and addressing disparities. J. Autism Dev. Disord. 2021, 51, 868-883. [CrossRef]

63. Magán-Maganto, M.; Bejarano-Martín, Á.; Fernández-Alvarez, C.; Narzisi, A.; García-Primo, P.; Kawa, R.; Posada, M.; Canal-Bedia, R. Early detection and intervention of ASD: A European overview. Brain Sci. 2017, 7, 159. [CrossRef]

64. Bishop-Fitzpatrick, L.; Smith DaWalt, L.; Greenberg, J.S.; Mailick, M.R. Participation in recreational activities buffers the impact of perceived stress on quality of life in adults with autism spectrum disorder. Autism Res. 2017, 10, 973-982. [CrossRef] [PubMed]

65. Anderson, S.R.; Romanczyk, R.G. Early intervention for young children with autism: Continuum-based behavioral models. J. Assoc. Pers. Sev. Handicap. 1999, 24, 162-173. [CrossRef]

66. Peters-Scheffer, N.; Didden, R.; Korzilius, H.; Sturmey, P. A meta-analytic study on the effectiveness of comprehensive ABA-based early intervention programs for children with autism spectrum disorders. Res. Autism Spectr. Disord. 2011, 5, 60-69. [CrossRef]

67. Gardiner, E.; Iarocci, G. Family quality of life and asd: The role of child adaptive functioning and behavior problems. Autism Res. 2015, 8, 199-213. [CrossRef]

68. Hou, Y.; Kim, S.Y.; Hazen, N.; Benner, A.D. Parents' perceived discrimination and adolescent adjustment in Chinese American families: Mediating family processes. Child Dev. 2017, 88, 317-331. [CrossRef] [PubMed]

69. Robert, M.; Leblanc, L.; Boyer, T. When satisfaction is not directly related to the support services received: Understanding parents' varied experiences with specialised services for children with developmental disabilities. Br. J. Learn. Disabil. 2015, 43, 168-177. [CrossRef]

70. Moh, T.A.; Magiati, I. Factors associated with parental stress and satisfaction during the process of diagnosis of children with autism spectrum disorders. Res. Autism Spectr. Disord. 2012, 6, 293-303. [CrossRef]

71. Wang, H.; Hu, X.; Han, Z.R. Parental stress, involvement, and family quality of life in mothers and fathers of children with autism spectrum disorder in mainland China: A dyadic analysis. Res. Dev. Disabil. 2020, 107, 103791. [CrossRef] 